УДК 622.276

ОПТИМИЗАЦИЯ РЕЖИМА СЖИГАНИЯ УГЛЕВОДОРОДНОГО ПОПУТНОГО ГАЗА В ФАКЕЛАХ

\title{
OPTIMIZATION OF COMBUSTION MODE OF HYDROCARBON ASSOCIATED GAS IN TORCHES
}

\author{
И.Х. Асадов
}

\section{Национальное аэрокосмическое агентство, г. Баку, Азербайджанская Республика}

\author{
Ilgar H. Asadov
}

\section{National Aerospace Agency, Baku, Azerbaijan Republic e-mail: asadzade@rambler.ru}

Аннотация. Статья посвящена вопросам оптимизации сжигания углеводородного попутного газа в факелах. Целью статьи является разработка нового метода для определения условий минимизации эмиссии сажи при сжигании углеводородного попутного газа. Основное внимание уделено вопросам аэрозольного загрязнения воздуха в зонах сжигания углеводородного попутного газа. Предлагается новый метод оптимизации, базирующийся на том факте, что объем эмитируемой сажи оказывается экстремальной функцией показателя Фрауде. Получено условие, при выполнении которого целевой функционал, характеризующий общее количество эмитируемого в атмосферу аэрозоля, достигает минимума. При этом обеспечивается минимально возможная среднеинтегральная величина аэрозольной эмиссии при факельном сжигании углеводородного попутного газа.

Представлены базовые сведения о методе Деличатсиоса, Макивена и Джонсона. Данный метод базируется на том факте, что объем эмитируемой сажи является экстремальной функцией показателя Фрауде. Показатель 
Фрауде является безразмерной величиной, характеризующей динамику развития пламени.

Разработан новый метод для определения условий минимизации эмиссии сажи при сжигании углеводородного попутного газа, основанный на нахождении экстремальной величины показателя Фрауде и обеспечивающий появление минимальной эмиссии аэрозоля.

Abstract. The article is devoted to the optimization of the combustion of hydrocarbon associated gas in flares. The purpose of the article is to develop a new method for determining the conditions for minimizing soot emission during combustion of hydrocarbon associated gas. The main attention is paid to the issues of aerosol air pollution in the zones of combustion of hydrocarbon associated gas. A new optimization method is proposed based on the fact that the volume of emitted soot turns out to be an extreme function of the Fraude exponent. A condition is obtained under which the target functional, which characterizes the total amount of aerosol emitted into the atmosphere, reaches a minimum. At the same time, the minimum possible average integral value of aerosol emission during flaring combustion of hydrocarbon associated gas is ensured.

Basic information about the method of Delichatsios, Machiven and Johnson is presented. This method is based on the fact that the volume of emitted soot is an extreme function of the Fraude exponent. The Fraude exponent is a dimensionless quantity that characterizes the dynamics of flame development.

A new method has been developed to determine the conditions for minimizing the emission of soot during the combustion of hydrocarbon associated gas, based on finding the extreme value of the Fraude exponent and ensuring the appearance of the minimum emission of aerosol.

Ключевые слова: попутный газ; аэрозоль; оптимизация; атмосфера; показатель Фрауде

Keywords: associated gas; spray can; optimization; atmosphere; Fraude exponent 


\section{Введение}

Сжигание попутного углеводородного газа является глобально используемым методом для удаления токсичного или коррозионного горючего газа путем превращения его в менее реактивные составляющие, реализуемым в зонах нефтепроизводства или нефтепереработки.

Согласно [1], только в 2008 г. в результате сжигания газа в атмосферу поступило 267.3 млн т $\mathrm{CO}_{2}$.

Контроль над объемами сжигаемого газа осуществляется спутниками DMSP, с помощью которых в 2012 г. обнаружены 7467 единиц факелов, сжигающих газ. Сжигание газа приводит к аэрозольному загрязнению воздуха, увеличению концентрации тяжелых металлов на поверхности близлежащих водоемов, повышает вероятность возникновения кислотных дождей и т.д. [1].

Далее в статье основное внимание будет уделено вопросам аэрозольного загрязнения воздуха в зонах сжигания углеводородного попутного газа.

В настоящей время имеются различные оценки эмиссии с факелов сжигания газа аэрозоля типа «черный углерод» (BC), или сажа. Так например, согласно [2], этот показатель равен 1.6 г/м³. Однако согласно [3],

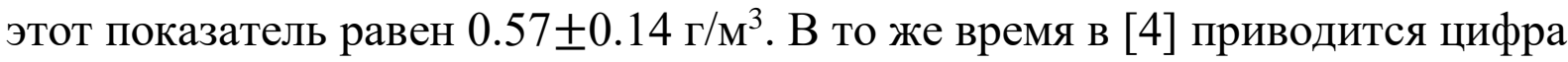
$0.28 \Gamma / \mathrm{M}^{3}$. Согласно [5], объем эмитируемого ВС прямо зависит от температуры факела, т.к. при высокой температуре происходит полное сгорание газа (рисунок 1).

Согласно [6], количество эмиссии аэрозоля в окружающую среду зависит от таких факторов, как отношение масса воздух / масса топлива; состав топливного газа; скорость подачи газа; эффективность сжигания; состояние внешней среды и др. Что касается эмитируемой при этом сажи, то этот тип аэрозоля является вторым по значимости после $\mathrm{CO}_{2}$ фактором, приводящим к появлению «парникового эффекта». 


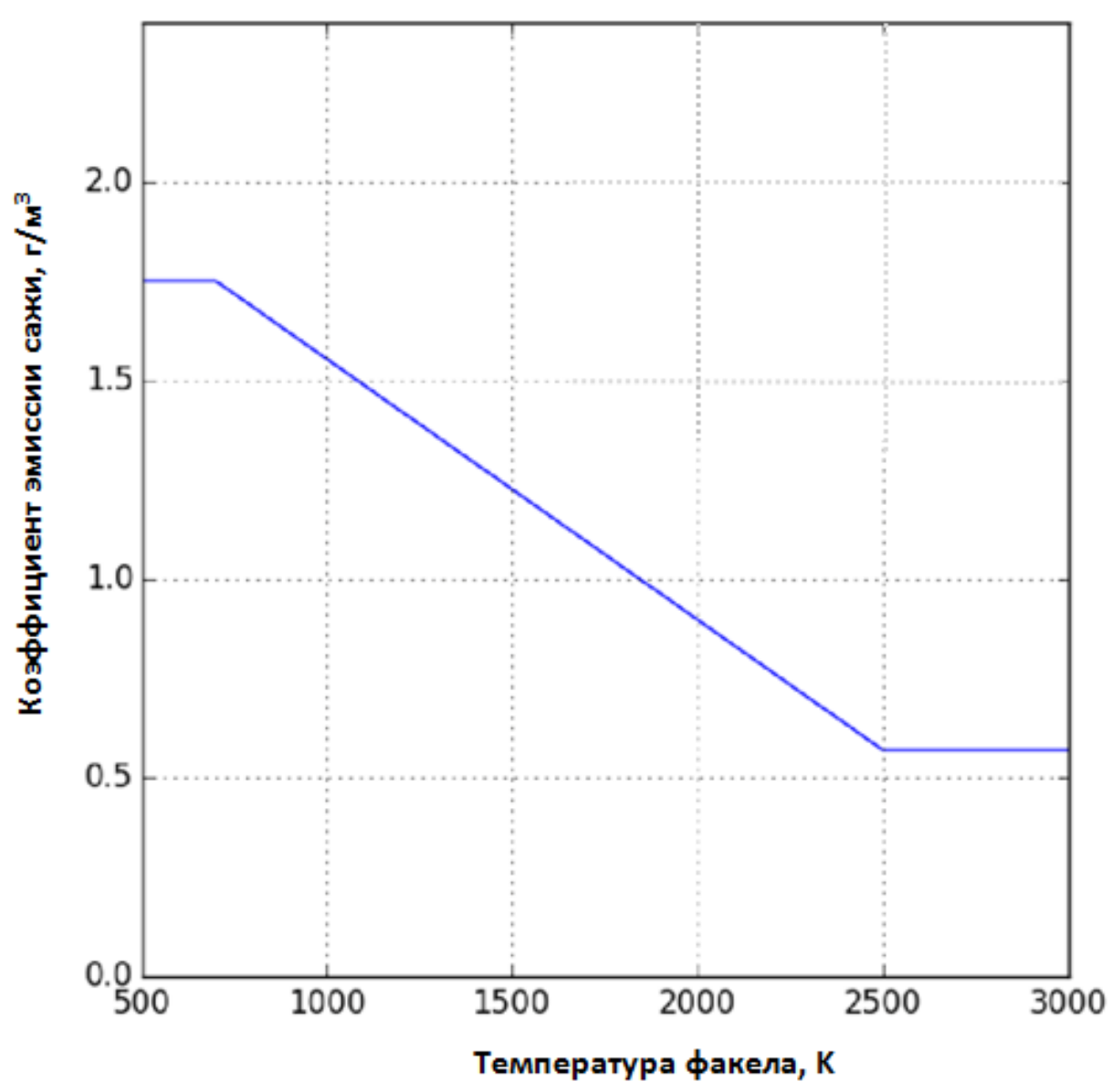

Рисунок 1. Зависимость объемов эмиссии ВС от температуры факела сжигания газа [5]

Целью настоящей статьи является разработка нового метода для определения условий минимизащии эмиссии сажи при сжигании углеводородного попутного газа.

Предлагаемый метод основывается на известном методе Деличатсиоса [7], Макивена и Джонсона [8], базирующемся на анализе показателя Фрауде. Вкратце рассмотрим основные положения этого метода.

\section{Базовые сведения о методе Деличатсиоса Макивена и Джонсона}

Данный метод базируется на том факте, что объем эмитируемой сажи является экстремальной функцией показателя Фрауде (рисунок 2). 


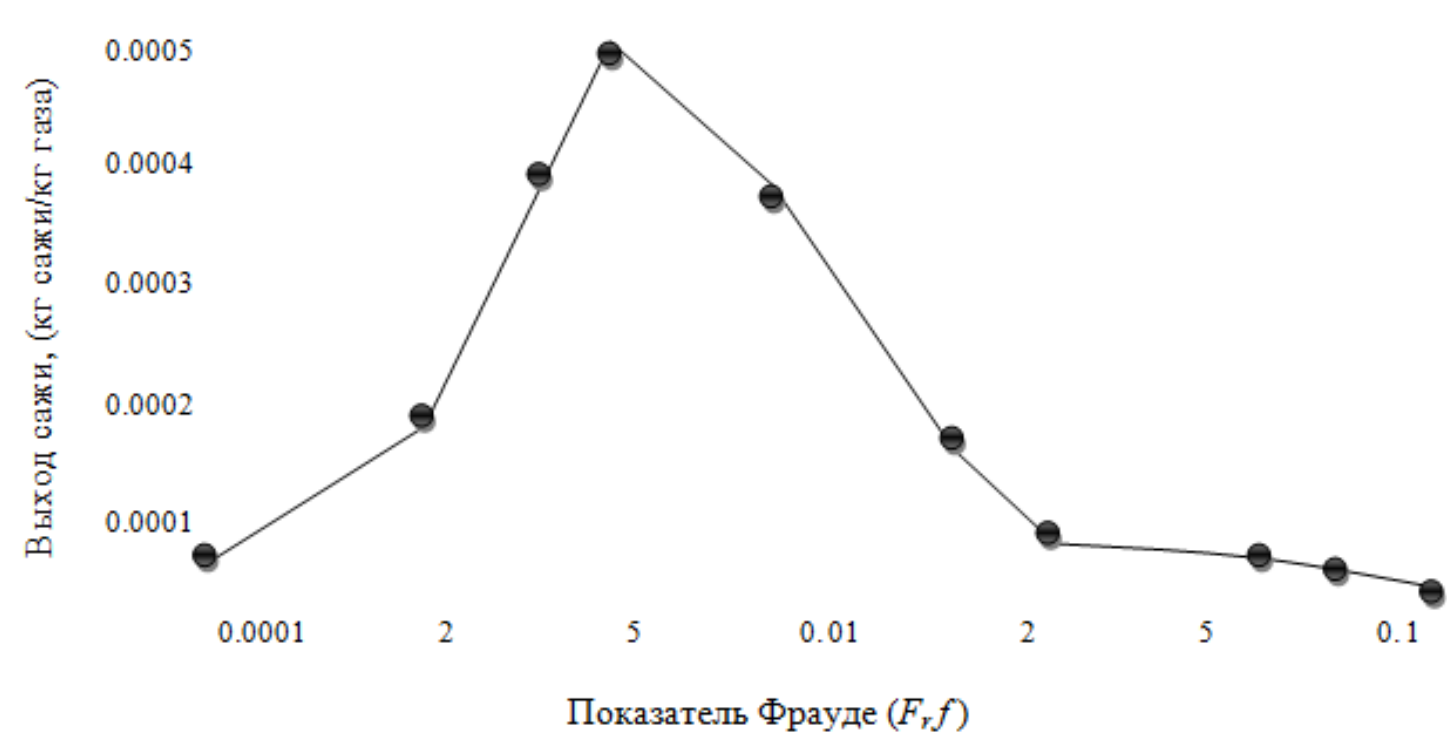

Рисунок 2. Экстремальное свойство функциональной зависимости объема эмиссии сажи при сжигании углеводородного попутного газа от значения показателя Фрауде [7]

Показатель Фрауде является безразмерной величиной, характеризующей динамику развития пламени и определяется следующим образом:

$$
F_{r}=\frac{V_{e} \cdot f_{s}^{\frac{3}{2}}}{\left(\frac{\Delta T_{f}}{T_{a m b}} g d_{e}\right)^{\frac{1}{2}} \cdot\left(\frac{\rho_{e}}{\rho_{a}}\right)^{\frac{1}{4}}},
$$

где

$$
\frac{1}{S+1}=\frac{\varphi}{\left(\frac{a}{f}\right)_{s}+\varphi}
$$

где $S$ - отношение масса воздуха / масса топлива;

$V_{e}-$ скорость подачи газа (м/с);

$T_{a m b}$ - температура окружающей среды, K;

$T_{\theta}$ - адиабатическая температура, $\mathrm{K}$;

$\Delta T_{f}$ - прирост температуры пламени, $\mathrm{K}$, определяемый как 


$$
\Delta \mathrm{T}_{\mathrm{f}}=\mathrm{T}_{\theta}-\mathrm{T}_{\mathrm{amb}}
$$

$g$ - ускорение свободного падения, м/ ${ }^{2}$;

$d_{e}$ - диаметр трубы, м;

$\rho_{\mathrm{a}}, \rho_{\mathrm{e}}-$ плотность воздуха и плотность сжигаемого газа, кг $/ \mathrm{M}^{3}$;

$\varphi$ - отношение эквивалентности, определяемое как

$$
\varphi=\left(\frac{A}{F}\right)_{S} /\left(\frac{A}{F}\right)_{a},
$$

где $\left(\frac{A}{F}\right)_{S}$ - стехиометрическая смесь воздух - газ, необходимая для полного сгорания газа;

$$
\begin{aligned}
& \left(\frac{A}{F}\right)_{a} \text { - фактическая величина смеси воздух - газ; } \\
& f_{s} \text { - фракция стехиометрической смеси. }
\end{aligned}
$$

На рисунке 3 приведены экспериментально снятые кривые объема эмиссии сажи от $F_{r}$ при разных значениях $d_{e}$.

Как видно из кривых, представленных на рисунке 3, диаметр трубы является существенным фактором, влияющим на объемы эмиссии сажи в атмосферу. В то же время, как видно из рисунков 2 и 3 , при малых и больших значениях $F_{r}$ следует ожидать меньших значений эмиссии сажи.

C учетом вышеизложенного предлагается метод нахождения экстремальной величины $F_{r}$, обеспечивающий появление минимальной эмиссии аэрозоля. 


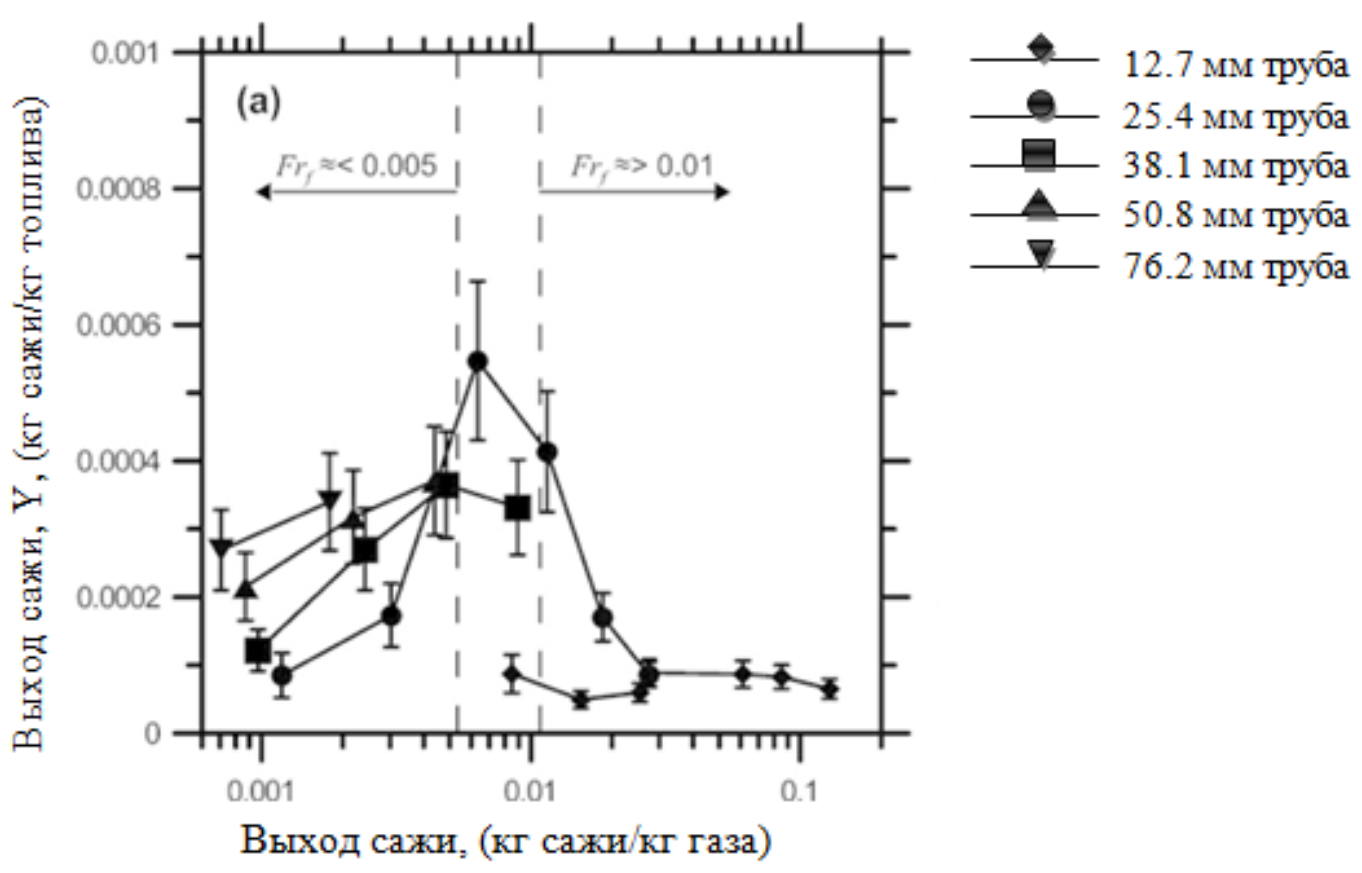

Рисунок 3. Экспериментально снятые кривые зависимости объема эмиссии сажи от значения показателя Фрауде при разных значениях $d_{e}$ [7]

\section{Предлагаемый метод}

Предлагаемый метод основывается на следующих положениях.

1. Наличие следующих множеств:

a) множество $D$, определяемое как

$$
D T=\left\{d_{e i}\right\}, i=\overline{1, n} ;
$$

b) множество $T$, определяемое как

$$
T=\left\{\Delta T_{f j}\right\}, j=\overline{1, n}
$$

2. Существование взаимно однозначной функциональной зависимости

$$
\Delta T_{f}=\varphi\left(d_{e}\right),
$$

физически означающей возможность выбора диаметра трубы в зависимости от значения показателя $\Delta T_{f}$. 
3. Допускаем, что функция $\varphi\left(d_{e}\right)$ удовлетворяет условию

$$
\sum_{i=1}^{n} \varphi\left(d_{e i}\right)=C ; C=\text { const }
$$

в дискретной записи и

$$
\int_{0}^{d_{\text {max }}} \phi\left(d_{e}\right) d\left(d_{e}\right)=C_{1} ; C_{1}=\text { const }
$$

в непрерывной форме записи.

4. Выдвигается требование приведения среднеинтегральной величины

$$
F_{r . c p}=\frac{1}{F_{r \max }} \int_{0}^{d_{e \max }} F_{r} d\left(d_{e}\right) .
$$

к экстремальной (максимум или минимум) величине. Для выполнения этого требования с учетом условия (6) должна быть вычислена оптимальная функция $\varphi\left(d_{e}\right)$, при которой $F_{r . c p} \rightarrow$ extr.

Проведем модельное исследование по реализации данного метода.

\section{Модельное исследование}

С учетом выражений (1), (6) и (7) составим целевой функционал вариационной оптимизации:

$$
\Phi=\int_{0}^{x_{\max }}\left[\frac{a_{1}}{\left[\frac{y(x) \cdot x}{a_{2}}\right]^{\frac{1}{2}} \cdot a_{3}}\right] d x+\lambda \int_{0}^{x_{m}} y(x) d x,
$$

где $a_{1}=V_{e} f_{S}^{\frac{2}{3}} ; a_{2}=\frac{T_{a m b}}{g} ; a_{3}=\frac{\rho_{e}}{\rho_{a}} ; x=d_{e} ; y=\Delta T_{f}$. 
Приняв $a_{4}=\frac{a_{1} \sqrt{a_{2}}}{a_{3}}$, функционал (8) перепишем как

$$
\Phi=\int_{0}^{d_{e_{\max }}} \frac{a_{4}}{\sqrt{y(x) x}} d x+\lambda \int_{0}^{d_{e \max }} y(x) d x
$$

Согласно [9], решение задачи $y(x)_{\text {opt }}$, при котором $\Phi \rightarrow$ extremum, должно удовлетворять условию

$$
-\frac{1}{2} \cdot \frac{a_{4}}{\sqrt{x}} \cdot y(x)^{-\frac{3}{2}}+\lambda=0
$$

Из (10) получим

$$
a_{4} y(x)^{\frac{3}{2}}=2 \lambda \cdot \sqrt{x}
$$

Из (11) находим

$$
y(x)=\sqrt{\left(\frac{2 \lambda \sqrt{x}}{a_{4}}\right)^{3}}=\lambda^{\frac{3}{2}} \sqrt{\frac{8 x^{3 / 2}}{a_{4}^{3}}} .
$$

С учетом выражений (6) имеем

$$
\int_{0}^{X_{m}} y(x) d x=C ; \quad C=\text { const } .
$$

Из (12) и (13) находим

$$
\lambda^{\frac{3}{2}} \int_{0}^{X_{m}} \sqrt{\frac{8 x^{\frac{3}{2}}}{a_{4}^{3}}} d x=C
$$


Из (14) имеем

$$
\lambda=\sqrt[3]{\left[\frac{C}{\left[\int_{0}^{x_{m}} \sqrt{\frac{8 x^{\frac{3}{2}}}{a_{4}^{3}}} d x\right]^{2}}\right.} .
$$

С учетом выражений (12) и (15) получим

$$
y(x)=\frac{C}{\int_{0}^{X_{m}} \sqrt{\frac{8 x^{\frac{3}{2}}}{a_{4}^{3}} d x}} \cdot \sqrt{\frac{8 x^{\frac{3}{2}}}{a_{4}^{3}}}=\frac{C \sqrt{x^{\frac{3}{2}}}}{\int_{0}^{X_{m}} \sqrt{x^{\frac{3}{2}}} d x} .
$$

Таким образом, при решении (16) целевой функционал (8) достигает экстремума. Для определения типа экстремума достаточно взять вторую производную интегранта в (8) по искомой функции. При этом полученный результат, указывающий на положительный знак второй производной (8) по искомой функции, свидетельствует о том, что функционал достигает своего минимума.

\section{Заключение}

Таким образом, с учетом выражения (16) можно утверждать, что при решении

$$
\Delta T_{f}=\frac{C \cdot \sqrt{d_{e}^{\frac{3}{2}}}}{\int_{0}^{X_{m}} \sqrt{d_{e^{\frac{3}{2}}}} d x}
$$

показатель $F_{r}$ достигает минимума. При этом согласно графику, показанному на рисунке 2, будет обеспечена минимально возможная 
среднеинтегральная величина аэрозольной эмиссии при факельном сжигании углеводородного попутного газа.

\section{Выводы}

Представлены базовые сведения о методе Деличатсиоса, Макивена и Джонсона. Данный метод базируется на том факте, что объем эмитируемой сажи является экстремальной функцией показателя Фрауде. Показатель Фрауде является безразмерной величиной, характеризующей динамику развития пламени.

Разработан новый метод для определения условий минимизации эмиссии сажи при сжигании углеводородного попутного газа, основанный на нахождении экстремальной величины показателя Фрауде и обеспечивающий появление минимальной эмиссии аэрозоля.

\section{Список используемых источников}

1. Deetz K., Vogel B. Development of a New Gas - Flaring Emission Dataset for Southern West Africa // Geoscientific Model Development. 2017. Vol. 10. Issue 4. P. 1607-1620. DOI: 10.5194/gmd-10-1607-2017.

2. Stohl A., Klimont Z., Eckhardt S., Kupiainen K., Shevcenko V.P., Kopeikin V.M., Novigatsky A.N. Black Carbon in the Arctic: the Underestimated Role of Gas Flaring and Residential Combustion Emissions // Atmospheric Chemistry and Physic. 2013. Vol. 13. P. 8833-8855. DOI: 10.5194/acp-13-8833-2013.

3. Schwarz J.P., Holloway J.S., Katich J.M., McKeen S., Kort E.A., Smith M.L., Ryerson T.B., Sweeney C., Peischl J. Black Carbon Emissions from the Bakken Oil and Gas Development Region // Environmental Science and Technology Letters. 2015. Vol. 2. Issue 10. P. 281-285. DOI: 10.1021/acs.estlett.5b00225. 
4. Johnson M.R., Devillers R.W., Thomson K.A. A Generalized Sky-LOSA Method to Quantify Soot/Black Carbon Emission Rates in Atmospheric Plumes of Gas Flares // Aerosol Science and Technology. 2012. Vol. 47. Issue 9. P. 10171029. DOI: 10.1080/02786826.2013.809401.

5. Caseiro A., Gehrke B., Rusker G., Leimbach D., Kaiser J.W. Gas Flaring Activity and Black Carbon Emissions in 2017 Derived from Sentinel-3A SLSTR // Earth System Science Data Discussions. 2019. URL: https://essd.copernicus.org/preprints/essd-2019-99/essd-2019-99.pdf (дата обращения: 25.04.2021). DOI: 10.5194/essd-2019-99.

6. Fawole O.G., Cai X.M., Nikolova I., McKenzine A.R. Self-Consistent Estimates of Emission Factors of Carboncontaining Pollutants from a Typical Gas Flare // Ife Journal o Science. 2020. Vol. 22. No. 2. P. 135-149. DOI: 10.4314/ijs.v22i2.13.

7. Delichatsios M. Transition from Momentum to Buoyancy-Controlled Turbulent Jet Diffusion Flames and Flame Height Relationships // Combustion and Flame. 1993. Vol. 92. Issue 4. P. 349-364.

8. McEwen J.D., Johnson M.R. Black Carbon Particulate Matter Emission Factors for Buoyancy - Driven Associated // Journal of the Air and Waste Management Association. 2012. Vol. 62. Issue 3. P. 307-321. DOI: 10.1080/10473289.2011.650040.

9. Эльсгольц Л.П. Дифференциальные уравнения и вариационное исчисление. М.: Наука, 1974. 432 с.

\section{References}

1. Deetz K., Vogel B. Development of a New Gas - Flaring Emission Dataset for Southern West Africa. Geoscientific Model Development, 2017, Vol. 10, Issue 4, pp. 1607-1620. DOI: 10.5194/gmd-10-1607-2017. 
2. Stohl A., Klimont Z., Eckhardt S., Kupiainen K., Shevcenko V.P., Kopeikin V.M., Novigatsky A.N. Black Carbon in the Arctic: the Underestimated Role of Gas Flaring and Residential Combustion Emissions. Atmospheric Chemistry and Physic, 2013, Vol. 13, pp. 8833-8855. DOI: 10.5194/acp-13-8833-2013.

3. Schwarz J.P., Holloway J.S., Katich J.M., McKeen S., Kort E.A., Smith M.L., Ryerson T.B., Sweeney C., Peischl J. Black Carbon Emissions from the Bakken Oil and Gas Development Region. Environmental Science and Technology Letters, 2015, Vol. 2, Issue 10, pp. 281-285. DOI: 10.1021/acs.estlett.5b00225.

4. Johnson M.R., Devillers R.W., Thomson K.A. A Generalized Sky-LOSA Method to Quantify Soot/Black Carbon Emission Rates in Atmospheric Plumes of Gas Flares. Aerosol Science and Technology, 2012, Vol. 47, Issue 9, pp. 10171029. DOI: 10.1080/02786826.2013.809401.

5. Caseiro A., Gehrke B., Rusker G., Leimbach D., Kaiser J.W. Gas Flaring Activity and Black Carbon Emissions in 2017 Derived from Sentinel-3A SLSTR. Earth System Science Data Discussions, 2019. URL: https://essd.copernicus.org/preprints/essd-2019-99/essd-2019-99.pdf (accessed 25.04.2021). DOI: 10.5194/essd-2019-99.

6. Fawole O.G., Cai X.M., Nikolova I., McKenzine A.R. Self-Consistent Estimates of Emission Factors of Carboncontaining Pollutants from a Typical Gas Flare. Ife Journal o Science, 2020, Vol. 22, No. 2, pp. 135-149. DOI: 10.4314/ijs.v22i2.13.

7. Delichatsios M. Transition from Momentum to Buoyancy-Controlled Turbulent Jet Diffusion Flames and Flame Height Relationships. Combustion and Flame, 1993, Vol. 92, Issue 4, pp. 349-364.

8. McEwen J.D., Johnson M.R. Black Carbon Particulate Matter Emission Factors for Buoyancy - Driven Associated. Journal of the Air and Waste Management Association, 2012, Vol. 62, Issue 3, pp. 307-321. DOI: 10.1080/10473289.2011.650040. 
9. Elsgolts L.P. Differentsial'nye uravneniya $i$ variatsionnoe ischislenie [Differential Equations and Calculus of Variations]. Moscow, Nauka Publ., 1974. 432 p. [in Russian].

\section{Сведения об авторе}

\section{About the Author}

Ильгар Хикмет оглы Асадов, аспирант Национального аэрокосмического агентства, г. Баку, Азербайджанская Республика

Ilgar H. Asadov, Post-graduate Student of National Aerospace Agency, Baku, Azerbaijan Republic

e-mail: asadzade@rambler.ru 